\title{
Long-time behavior of scalar conservation laws with critical dissipation
}

\author{
Dallas Albritton and Rajendra Beekie
}

\begin{abstract}
The critical Burgers equation $\partial_{t} u+u \partial_{x} u+\Lambda u=0$ is a toy model for the competition between transport and diffusion with regard to shock formation in fluids. It is well known that smooth initial data does not generate shocks in finite time. Less is known about the long-time behavior for 'shock-like' initial data: $u_{0} \rightarrow \pm a$ as $x \rightarrow \mp \infty$. We describe this long-time behavior in the general setting of multidimensional critical scalar conservation laws $\partial_{t} u+\operatorname{div} f(u)+\Lambda u=0$ when the initial data has limits at infinity. The asymptotics are given by certain self-similar solutions, whose stability we demonstrate with the optimal diffusive rates.
\end{abstract}

\section{Introduction}

Our motivating example is the Burgers equation with critical non-local dissipation

$$
\partial_{t} u+u \partial_{x} u+\Lambda u=0
$$

and 'shock-like' initial data:

$$
u_{0}(x) \rightarrow \pm a \quad \text { as } x \rightarrow \mp \infty
$$

where $\Lambda=(-\Delta)^{1 / 2}$ and $a>0$. This equation arises as a toy model in fluid mechanics. It models the competition between the transport non-linearity $u \partial_{x} u$, which drives the solution towards a shock, and the dissipation term $\Lambda u$, whose smoothing effects counteract the tendency of the non-linearity to form shocks. The equation is critical in the sense that these two terms are in balance. In PDE terms, the strongest known monotone quantities, the $L^{\infty}$ norm and total variation, are invariant under the scaling symmetry

$$
u \rightarrow u(\lambda x, \lambda t)
$$

which preserves the equation (1.1).

By now, it is well known that solutions of (1.1) evolving from smooth initial data do not form shocks in finite time. What happens in infinite time? We answer this question for 
the critical Burgers equation (1.1) and in the more general context of scalar conservation laws with critical non-local dissipation in $\mathbb{R}^{n}$ :

$$
\partial_{t} u+\operatorname{div} f(u)+\Lambda u=0,
$$

where the initial data has 'limits at infinity'. The long-time behavior is described to leading order by certain self-similar solutions, that is, solutions invariant under the scaling symmetry (1.3).

Let $h \in C^{\infty}\left(S^{n-1}\right)$ and $u_{0}^{\text {ss }}(x)=h(x /|x|)$. Let $f: \mathbb{R} \rightarrow \mathbb{R}^{n}$ belong to $C_{\text {loc }}^{\infty}\left(\mathbb{R} ; \mathbb{R}^{n}\right)$. Let $v_{0} \in L^{\infty}\left(\mathbb{R}^{n}\right)$ with $\left|v_{0}\right| \rightarrow 0$ as $|x| \rightarrow+\infty$, specifically,

$$
\|v\|_{L^{\infty}\left(\mathbb{R}^{n} \backslash B_{R}\right)} \rightarrow 0 \quad \text { as } R \rightarrow+\infty .
$$

Let $u_{0}=u_{0}^{\mathrm{ss}}+v_{0}$ and $\left\|u_{0}^{\mathrm{ss}}\right\|_{L^{\infty}\left(\mathbb{R}^{n}\right)},\left\|u_{0}\right\|_{L^{\infty}\left(\mathbb{R}^{n}\right)} \leq m$. Let $u^{\mathrm{ss}}, u$ be the unique entropy solutions to (1.4) with initial data $u_{0}^{\mathrm{ss}}, u_{0}$, respectively. In the context of (1.4), the notion of entropy solution was introduced by Alibaud in [3], and we review it below. Notice that, by virtue of its uniqueness, $u^{\text {ss }}$ must be self similar.

Here is our main theorem:

Theorem 1.1 (Long-time behavior). The above entropy solution u converges to the selfsimilar solution $u^{\mathrm{ss}}$ with the following (diffusive) rates:

$$
\left\|u(\cdot, t)-u^{\mathrm{ss}}(\cdot, t)\right\|_{L^{q}\left(\mathbb{R}^{n}\right)} \lesssim_{m, n} o_{t \rightarrow+\infty}(1) t^{\frac{n}{q}-\frac{n}{p}}\left\|u_{0}-u_{0}^{\mathrm{ss}}\right\|_{L^{p}\left(\mathbb{R}^{n}\right)}
$$

for all $1<p \leq q \leq+\infty$. If $p=1$, then (1.6) holds with $O(1)$ instead of $o(1)$ on the right-hand side.

When $f \equiv 0,(1.4)$ reduces to the fractional heat equation, and the above diffusive rates are easily seen to be sharp.

In dimension $n=1$, we also have stability in BV:

Theorem 1.2 (BV convergence). If also $u_{0} \in \mathrm{BV}(\mathbb{R})$, then

$$
\left\|u(\cdot, t)-u^{\mathrm{ss}}(\cdot, t)\right\|_{\mathrm{TV}\left(\mathbb{R}^{n}\right)} \rightarrow 0 \quad \text { as } t \rightarrow+\infty .
$$

Additionally, $u^{\mathrm{ss}}$ is monotone and satisfies the following spatial asymptotics:

$$
C^{-1}\langle x\rangle^{-1} \leq\left|u^{\mathrm{ss}}(x, 1)-u_{0}^{\mathrm{ss}}\right| \leq C\langle x\rangle^{-1}, \quad|x| \geq C,
$$

provided that $u_{0}^{\text {ss }}$ is not identically constant. ${ }^{1}$ Notice that, when $n \geq 2, u_{0}^{\text {ss }}$ does not generally belong to $\mathrm{BV}\left(\mathbb{R}^{n}\right)$, and the total variation is no longer scaling invariant.

\footnotetext{
${ }^{1}$ It may be possible to obtain more precise spatial asymptotics for $u^{\mathrm{ss}}$ and its derivatives by analyzing the similarity profile $u^{\text {ss }}(\cdot, 1)$, which satisfies a quasilinear non-local elliptic equation.

Added in print: In the rarefaction case, qualitative properties of the self-similar profile (symmetry, monotonicity, and convexity of $u^{\mathrm{ss}}(\cdot, 1)$, as well as asymptotics for $\partial_{x} u^{\mathrm{ss}}(x, 1)$ as $\left.|x| \rightarrow+\infty\right)$ were studied in Theorem 1.7 of [5] from this perspective.
} 


\subsection{Comparison with existing literature}

The critical Burgers equation (1.1) belongs to the following family of Burgers equations with fractional diffusion:

$$
\partial_{t} u+u \partial_{x} u+\Lambda^{s} u=0,
$$

where $s \in(0,2]$. These models were considered by Biler, Funaki, and Woyczynski in [7], where they are known as fractal Burgers equations. One may consider also the analogous conservation laws with fractional diffusion $\Lambda^{s}$. The relevant literature is fairly extensive:

Regularity theory. A detailed picture of the regularity theory of (1.9) was shown by Kiselev, Nazarov, and Shterenberg in [32] in the periodic setting. When $s \geq 1$, smooth initial data gives global smooth solutions, whereas when $s<1$, solutions may develop shocks in finite time. In that case, solutions may be continued uniquely within the class of entropy solutions. See [4,20] for further discussions on regularity vs. blow-up. The proof of global regularity in [32] in the critical case follows the method of 'moduli of continuity'. This method was introduced in [33] by Kiselev, Nazarov, and Volberg in the context of the critical SQG equation: ${ }^{2}$

$$
\partial_{t} \theta+\vec{R}^{\perp} \theta \cdot \nabla \theta+\Lambda \theta=0 .
$$

Other proofs of the regularity of (SQG) are contained in [11] (De Giorgi's method), [31], [34] (Nash's method), [17] ('nonlinear maximum principle'), and [16]. The above proofs can be categorized as proofs of smoothing $[11,34]$ or propagation of regularity $[16,17$, $31,33]$. The smoothing proofs notably 'forget' that the equation is nonlinear. Alternative proofs of regularity for (1.1), based on smoothing, were given in [12] (De Giorgi's method) and $[38,39]$ (non-divergence form techniques). We rely on these smoothing estimates, particularly those of Silvestre, in an essential way below. ${ }^{3}$

Long-time behavior. The long-time behavior of (1.9) is perhaps less well studied than its regularity. When $s \in(0,2)$ and the initial data is well localized, the non-linearity of $(1.9)$ is 'irrelevant', in the sense of [9], for the long-time dynamics. When $s=1$, Iwabuchi [24,25] demonstrated that all solutions with $u_{0} \in L^{1} \cap \dot{B}_{\infty, 1}^{0}$ converge to the Poisson kernel. When $s=2$, the spaces $L^{1}$ and $\mathcal{M}$ (finite measures) are critical, and it is classical that the long-time behavior is given by a self-similar solution, sometimes called a diffusion wave. This case and its precise asymptotic behavior can be illuminated by the Cole-Hopf transformation [6, 14, 29, 35].

What about non-decaying solutions? The current best results in this direction concern 'rarefaction-like' initial data, that is, $a<0$ in (1.2). In [28], it was shown that such solutions converge to an inviscid rarefaction wave when $s>1$. In [5], it was shown that when

\footnotetext{
${ }^{2}$ This method has since been generalized to other models, including the one-dimensional critical Keller-Segel equations [10] and the one-dimensional fractional Euler alignment system [19].

${ }^{3}$ For supercritical SQG, global regularity remains open, though it is possible to show eventual regularity $[13,18,30,37]$. We mention also the recent extension of [11] to bounded domains in [41].
} 
$s=1$, the solutions converge to a certain self-similar solution, and when $s<1$, the nonlinearity is 'irrelevant' in the long-time asymptotic expansion. Notably, in the rarefaction case, the potential term in the energy estimates for the linearized equation appears with a good sign.

In this paper, we analyze the case of 'shock-like' initial data, which is less clear. Initially, one might wonder whether (i) solutions converge to a smooth traveling or standing wave, known as a 'viscous shock', or perhaps (ii) solutions form a shock in infinite time. Regarding (i), it was already shown in [7] that traveling wave solutions satisfying reasonable regularity conditions do not exist when $s \in(0,1]$. Regarding (ii), one might additionally wonder whether the standing waves constructed in the subcritical case $s>1$ in [15] converge to a shock as $s \rightarrow 1^{+}$. This is apparently also not the case, as we show in Theorem 1.1.

It is tempting to conjecture that, in the subcritical case $s>1$, shock-like solutions of (1.9) behave as in the classical case $s=2$, where there is a unique viscous shock, whose global asymptotic stability was shown by Ilín and Oleĭnik in [23]. See [27, 36, 44] and many others for further developments and precise asymptotics. For $s \in(1,2)$, the uniqueness, spatial asymptotics, and global asymptotic stability of the monotone viscous shocks constructed in [15] do not seem to have appeared in the literature, although local asymptotic stability was recently demonstrated in [1].

Self-similarity and (non-)uniqueness. The two-dimensional Navier-Stokes equations

$$
\partial_{t} \omega+u \cdot \nabla \omega-\Delta \omega=0, \quad u=\nabla^{\perp} \Delta^{-1} \omega
$$

exhibit a family of self-similar solutions known as the Oseen vortices: $\omega(x, t)=$ $\alpha \Gamma(x, t / v)$, where $\Gamma$ is the heat kernel and $\alpha=\int \omega_{0} d x$ is the circulation. In [21], Gallay and Wayne famously showed that all localized solutions converge to Oseen vortices as $t \rightarrow+\infty$, and, moreover, the vortex solutions are unique within a natural solution class. Our situation is analogous, with the circulation $\alpha$ corresponding to the jump parameter $a$ in (1.2). By contrast, self-similar solutions of the three-dimensional Navier-Stokes equations are expected to be non-unique [22,26]. For (SQG), this is investigated in forthcoming work of Bradshaw and the first author. While the entropy solutions of (1.1) are unique, there may be a different class of self-similar solutions with potential non-uniqueness, for example, with $u_{0} \sim \log x$, so that $\nabla u_{0}$ is -1 -homogeneous.

\subsection{Main idea}

Our starting point is the existence and uniqueness of $L^{\infty}$ entropy solutions to (1.4), due to Alibaud [3], which immediately gives the existence and uniqueness of a self-similar solution $u^{\text {ss }}$. Let $v=u-u^{\text {ss }}$ be the difference between an entropy solution $u$ and the selfsimilar solution. Consider a sequence $\left(v^{(k)}\right)_{k \in \mathbb{N}}$ obtained by 'zooming out' on $v$ using the scaling symmetry (1.3). Then establishing $v(\cdot, t) \rightarrow 0$ as $t \rightarrow+\infty$ is the same as establishing $v^{(k)} \rightarrow 0$ on $\mathbb{R}^{n} \times(1 / 2,1)$ as $k \rightarrow+\infty$. To analyze the new problem, we exploit a key (standard) observation about viscous scalar conservation laws, namely, that 
$v$ satisfies the viscous continuity equation

$$
\partial_{t} v+\operatorname{div}(g v)+\Lambda v=0
$$

where

$$
g=\frac{f(u)-f\left(u^{\mathrm{ss}}\right)}{u-u^{\mathrm{ss}}} \in L^{\infty}\left(\mathbb{R}^{n} \times(0,+\infty)\right) .
$$

In our setting, the main difficulty is that at the initial time, $g$ is no better than bounded, since $u_{0}^{\mathrm{ss}}$ is not continuous. This is an essential feature of the problem, and we handle it using two tools:

(1) smoothing for drift-diffusion equations. By the known regularity theory, the solution, which is initially merely bounded, instantaneously becomes $C^{\alpha}$-in- $x$. This may be bootstrapped to higher regularity. The key point is then to move the problem past $t=0$, which is done by the

(2) controlled speed of propagation. Solutions of (1.10) have finite propagation speed up to the effect of the diffusion. This allows us to keep the initial spatial decay of the solution for small positive times and exploit (1.10) with smooth coefficients and smooth, decaying initial data.

The above tools, due to [38,39] and [3], respectively, are key to our arguments. We encounter a further, technical difficulty in that the controlled speed of propagation only allows us to propagate $L^{1}$-based quantities. This requires the use of special norms $\|\cdot\|_{\ell_{k}^{q} L_{x}^{p}\left(\mathbb{R}^{n}\right)}$, for example,

$$
\|v\|_{\ell_{k}^{\infty} L_{x}^{1}\left(\mathbb{R}^{n}\right)}=\sup _{k \in \mathbb{Z}^{n}} \int_{k+(-1 / 2,1 / 2)^{n}}|v(x)| d x .
$$

After the initial time, we use the smoothing effect to estimate more standard quantities, such as $\|v\|_{L^{q}\left(\mathbb{R}^{n}\right)}$, in terms of these special norms. ${ }^{4}$ For this, we use pointwise estimates for fundamental solutions of non-local parabolic equations with subcritical lower order terms, due to Xie and Zhang [43]. When $f$ is merely Lipschitz, we offer less precise asymptotics, see Remark 3.3.5

\section{Preliminaries}

In the sequel, constants in the $C, \lesssim$ notation may implicitly depend on $n \geq 1, f \in$ $W_{\text {loc }}^{1, \infty}\left(\mathbb{R} ; \mathbb{R}^{n}\right)$.

\footnotetext{
${ }^{4}$ Similar norms appear in the Navier-Stokes literature. See [8] and the references therein. Apparently, these spaces are known as Wiener amalgam spaces.

${ }^{5}$ Added in print: See Remark 3.2 for an alternative proof, due to Hongjie Dong, based on a maximal function estimate.
} 
Recall that the Poisson kernel $P$ is given by

$$
P(x, t)=c_{n} \frac{t}{\left(|x|^{2}+t^{2}\right)^{\frac{n+1}{2}}},
$$

where $c_{n}>0$ is chosen to satisfy $\int P(x, t) d x=1$ for all $t>0$.

In [3, Definition 2.3], Alibaud introduced the notion of entropy solution to the critical scalar conservation law (1.4). We summarize only the facts we need about entropy solutions; see [3, Section 3]. For each $u_{0} \in L^{\infty}\left(\mathbb{R}^{n}\right)$, there exists a unique entropy solution $u$ of (1.4). This solution exists globally and satisfies the maximum principle

$$
\|u\|_{L_{t, x}^{\infty}\left(\mathbb{R}^{n} \times(0,+\infty)\right)} \leq\left\|u_{0}\right\|_{L^{\infty}\left(\mathbb{R}^{n}\right)}
$$

The PDE (1.4) is satisfied in the distributional sense. Finally, $u$ belongs to the space $C\left([0, T] ; L^{1}(K)\right)$ for each $T>0$ and compact $K \subset \mathbb{R}^{n}$.

The following proposition is contained in [3, Theorem 3.2]:

Proposition 2.1 (Controlled speed of propagation). Let $u_{0}, \tilde{u}_{0} \in L^{\infty}\left(\mathbb{R}^{n}\right)$. Consider $u, \tilde{u}$ entropy solutions to (1.4) with initial conditions $u_{0}$ and $\tilde{u}_{0}$, respectively. Then for all $x_{0} \in \mathbb{R}^{n}$, all $t>0$ and all $R>0$,

$$
\int_{B\left(x_{0}, R\right)}|u(x, t)-\tilde{u}(x, t)| d x \leq \int_{B\left(x_{0}, R+L t\right)} P(\cdot, t) *\left|u_{0}-\tilde{u}_{0}\right| d x,
$$

where $L$ is the Lipschitz constant of $f$ on $[-m, m]$ and $m$ is defined by $m=$ $\max \left(\left\|u_{0}\right\|_{L^{\infty}\left(\mathbb{R}^{n}\right)},\left\|\tilde{u}_{0}\right\|_{L^{\infty}\left(\mathbb{R}^{n}\right)}\right)$.

We use Proposition 2.1 to establish the following corollary.

Proposition 2.2 (Controlled $\mathrm{BV}$ ). If $u_{0} \in \mathrm{BV}\left(\mathbb{R}^{n}\right)$, then $u(\cdot, t) \in \mathrm{BV}\left(\mathbb{R}^{n}\right)$ with $\|u(\cdot, t)\|_{\mathrm{TV}\left(\mathbb{R}^{n}\right)} \leq\left\|u_{0}\right\|_{\mathrm{TV}\left(\mathbb{R}^{n}\right)}$ for all $t>0$. Let $\psi \in C_{0}^{\infty}\left(\mathbb{R}^{n}\right)$ be non-negative and radial with $\psi \equiv 1$ in a neighborhood of the origin. Let $\psi(x, t)=\psi(x-x L t /|x|)$ when $|x| \geq L t$ and $\psi \equiv 1$ otherwise. Then, for all $x_{0} \in \mathbb{R}^{n}$, all $t>0$, and $k=1, \ldots, n$,

$$
\int_{\mathbb{R}^{n}} \psi\left(x-x_{0}\right)\left|\omega_{k}(x, t)\right| d x \leq \int_{\mathbb{R}^{n}} \psi\left(x-x_{0}, t\right) P(\cdot, t) *\left|\omega_{k, 0}\right| d x,
$$

where $\omega_{k}=\partial_{k} u$ and $\omega_{k, 0}=\partial_{k} \omega_{0}$ are finite measures. ${ }^{6}$

By approximation, if also $\nabla u(\cdot, t) \in L^{1}\left(\mathbb{R}^{n}\right)$ for a given $t>0$, then $\psi=\mathbf{1}_{B_{R}}$ with $R>0$ is an admissible weight function.

Proof. The global BV bound is directly from [3, Proposition 3.4]. Let us justify (2.4) with $x_{0}=0$ when $\nabla u_{0} \in L^{1}\left(\mathbb{R}^{n}\right)$ is compactly supported. First, Alibaud's formula (2.3) holds with integration against weight $\psi$ as in (2.4). This is shown by integrating (2.3)

${ }^{6}$ This is why we require integration against continuous $\psi$ on the left-hand side. 
according to the principle $\int_{\mathbb{R}^{n}} \psi F d x=\int_{0}^{\infty} \int_{\{\psi>\lambda\}} F d x d \lambda$. Let $D_{k}^{\varepsilon}$ be the different quotient operator $D_{k}^{\varepsilon} u=\left(u(x)-u\left(x-\varepsilon \vec{e}_{k}\right)\right) / \varepsilon$. Letting $\tilde{u}=u\left(\cdot-\varepsilon \vec{e}_{k}, t\right)$ in (2.3) with weight $\psi$, and dividing by $\varepsilon$, we have

$$
\int_{\mathbb{R}^{n}} \psi(x)\left|D_{k}^{\varepsilon} u(x, t)\right| d x \leq \int_{\mathbb{R}^{n}} \psi(x, t) P(\cdot, t) *\left|D_{k}^{\varepsilon} u_{0}\right| d x .
$$

We have $D_{k}^{\varepsilon} u_{0} \rightarrow \omega_{k, 0}$ strongly in $L^{1}\left(\mathbb{R}^{n}\right)$. Then $P(\cdot, t) *\left|D_{k}^{\varepsilon} u_{0}\right| \rightarrow P(\cdot, t) *\left|\omega_{k, 0}\right|$ in $L^{1}\left(\mathbb{R}^{n}\right)$ also. This implies that the left-hand side remains bounded as $\varepsilon \rightarrow 0^{+}$. Hence, $\nabla u(\cdot, t)$ actually belongs to $L^{1}\left(\mathbb{R}^{n}\right)$, and the left-hand side converges to $\int_{\mathbb{R}^{n}} \psi(x)\left|\omega_{k}(x, t)\right| d x$ as $\varepsilon \rightarrow 0^{+}$. To complete the proof for general $u_{0} \in \mathrm{BV}\left(\mathbb{R}^{n}\right)$, we approximate $u_{0}$ in $L^{\infty}\left(\mathbb{R}^{n}\right)$ by $u_{0}^{(i)}, i \in \mathbb{N}$, and we approximate $\nabla u_{0}$ weakly-* in $\mathcal{M}\left(\mathbb{R}^{n}\right)$ by $\nabla u_{0}^{(i)}$ compactly supported, belonging to $L^{1}\left(\mathbb{R}^{n}\right)$, and satisfying $\left|\omega_{k, 0}^{(i)}\right| \stackrel{*}{\rightarrow}\left|\omega_{k, 0}\right|$ in the sense of measures. Then one may verify, using the Lebesgue dominated convergence theorem and kernel estimates, that $P(\cdot, t) *\left|\omega_{k, 0}^{(i)}\right| \rightarrow P(\cdot, t) *\left|\omega_{k, 0}\right|$ strongly in $L^{1}\left(\mathbb{R}^{n}\right)$. The left-hand side is handled by lower semicontinuity. This completes the proof.

Proposition 2.1 only allows us to propagate $L^{1}$-based quantities, which then smooth to $L^{q}$-based quantities, $q \geq 1$, after the initial time:

Let $\ell>0$ and $\ell \square(k)$ be the open cube with center at $k$ and side length $\ell$. That is, $\ell \square(k)=k+(-\ell / 2, \ell / 2)^{n}$. We write $\square(k)=1 \square(k)$. Define

$$
\|f\|_{\ell_{k}^{p} L_{x}^{q}\left(\mathbb{R}^{n}\right)}=\|\| f\left\|_{L_{x}^{q}(\square(k))}\right\|_{\ell_{k}^{p}\left(\mathbb{Z}^{n}\right)} .
$$

When $p=+\infty$, the space $\ell_{k}^{\infty} L_{x}^{q}\left(\mathbb{R}^{n}\right)$ is known in the literature as $L_{\text {uloc }}^{q}\left(\mathbb{R}^{n}\right)$. We have $L^{p}\left(\mathbb{R}^{n}\right)=\ell_{k}^{p} L_{x}^{p}\left(\mathbb{R}^{n}\right)$ with equality of norms. We also have the obvious embeddings

$$
\|f\|_{\ell_{k}^{p} L_{x}^{q_{1}}\left(\mathbb{R}^{n}\right)} \leq\|f\|_{\ell_{k}^{p} L_{x}^{q_{2}}\left(\mathbb{R}^{n}\right)}
$$

when $q_{1} \leq q_{2}$, and

$$
\|f\|_{\ell_{k}^{p_{2}} L_{x}^{q}\left(\mathbb{R}^{n}\right)} \leq\|f\|_{\ell_{k}^{p_{1}} L_{x}^{q}\left(\mathbb{R}^{n}\right)}
$$

when $p_{1} \leq p_{2}$. The short-time and small-distance behavior of these spaces is akin to that of $L^{q}\left(\mathbb{R}^{n}\right)$, whereas the large-distance behavior is more closely akin to that of $L^{p}\left(\mathbb{R}^{n}\right)$.

We will require the following smoothing estimates when $q=1$ or $p=q$. However, it is no more effort to prove the general estimates:

Lemma 2.3 (Smoothing for the heat equation). Let $p, q_{1}, q_{2} \in[1,+\infty]$ with $q_{1} \leq q_{2}$. Let $w_{0} \in \ell_{k}^{p} L_{x}^{q_{1}}\left(\mathbb{R}^{n}\right)$. Define

$$
w(\cdot, t)=P(\cdot, t) * w_{0}
$$

Then for $t \leq 1$,

$$
\|w(\cdot, t)\|_{\ell_{k}^{p} L_{x}^{q_{2}}\left(\mathbb{R}^{n}\right)} \lesssim t^{\frac{n}{q_{2}}-\frac{n}{q_{1}}}\left\|w_{0}\right\|_{\ell_{k}^{p} L_{x}^{q_{1}}\left(\mathbb{R}^{n}\right)} .
$$


Proof. Let $k \in \mathbb{Z}^{n}$. We decompose $\mathbb{R}^{n}$ into near-to- $k$ and far-from- $k$ regions:

$$
\begin{gathered}
\|w(x, t)\|_{L_{x}^{q_{2}(\square(k))} \leq} \leq \underbrace{\left\|\int_{y \in 3 \square(k)} P(x-y, t)\left|w_{0}\right|(y) d y\right\|_{L_{x}^{q_{2}(\square(k))}}}_{=I_{1}(k)} \\
+\underbrace{\left\|\int_{y \in \mathbb{R}^{n} \backslash 3 \square(k)} P(x-y, t)\left|w_{0}\right|(y) d y\right\|_{L_{x}^{q_{2}(\square(k))}}}_{=I_{2}(k)} .
\end{gathered}
$$

Eventually, we will sum in $\ell_{k}^{p}\left(\mathbb{Z}^{n}\right)$. First, we estimate $I_{1}(k)$ :

$$
I_{1}(k) \leq\left\|\sum_{|j|_{\infty} \leq 1} P(\cdot, t) *\left(\mathbf{1}_{\square(k+j)}\left|w_{0}\right|\right)\right\|_{L_{x}^{q_{2}}\left(\mathbb{R}^{n}\right)} \lesssim t^{\frac{n}{q_{2}}-\frac{n}{q_{1}}} \sum_{|j|_{\infty} \leq 1}\left\|w_{0}\right\|_{L_{x}^{q_{1}}(\square(k+j))},
$$

by Young's convolution inequality. We now sum in $\ell_{k}^{p}\left(\mathbb{Z}^{n}\right)$. By the triangle inequality, and since there are only a finite number of boxes (specifically, $3^{n}$ ) in the $j$ sum, we have

$$
\left\|I_{1}(k)\right\|_{\ell_{k}^{p}\left(\mathbb{Z}^{n}\right)} \lesssim t^{\frac{n}{q_{2}}-\frac{n}{q_{1}}}\left\|w_{0}\right\|_{\ell_{k}^{p} L_{x}^{q_{1}}\left(\mathbb{R}^{n}\right)} .
$$

Now we estimate $I_{2}(k)$ :

$$
\begin{aligned}
I_{2}(k) & \leq \sum_{|j|_{\infty}>1} \int_{\square(k-j)}\left|w_{0}(y)\right|\|P(x-y, t)\|_{L_{x}^{q_{2}}(\square(k))} d y \\
& \leq \sum_{|j|_{\infty}>1}\left[\sup _{x \in \square(k)} \sup _{y \in \square(k-j)} P(x-y, t)\right] \int_{\square(k-j)}\left|w_{0}(y)\right| d y,
\end{aligned}
$$

where we used Hölder's inequality in $x$ and $|\square(k)|=1$. When $x \in \square(k)$ and $y \in$ $\square(k-j)$, we have $|x-y| \geq|j|-1$. Recall that $P(z, t) \lesssim t /|z|^{n+1} \lesssim 1 /|z|^{n+1}$ for $t \leq 1$. Hence,

$$
\sup _{x \in \square(k)} \sup _{y \in \square(k-j)} P(x-y, t) \lesssim \frac{1}{(|j|-1)^{n+1}},
$$

and

$$
I_{2}(k) \lesssim \sum_{|j|_{\infty}>1} \frac{1}{(|j|-1)^{n+1}} \int_{\square(k-j)}\left|w_{0}(y)\right| d y .
$$

One may recognize (2.16) as a discrete convolution with a summable-in- $j$ kernel. Applying $\|\cdot\|_{\ell_{k}^{p}\left(\mathbb{Z}^{n}\right)}$, we have

$$
\left\|I_{2}(k)\right\|_{\ell_{k}^{p}\left(\mathbb{Z}^{n}\right)} \lesssim \sum_{|j|_{\infty}>1} \frac{1}{(|j|-1)^{n+1}}\left\|w_{0}\right\|_{\ell_{k}^{p} L_{x}^{1}\left(\mathbb{R}^{n}\right)} \lesssim\left\|w_{0}\right\|_{\ell_{k}^{p} L_{x}^{q_{1}}\left(\mathbb{R}^{n}\right)},
$$

where we used the trivial embedding (2.7). This completes the proof.

We now justify that the entropy solutions immediately become Hölder continuous and better: 
Proposition 2.4 (Regularity). Let $u$ be the unique entropy solution of (1.4) with initial data satisfying $\left\|u_{0}\right\|_{L^{\infty}\left(\mathbb{R}^{n}\right)} \leq m$. Suppose also that $f \in C_{\mathrm{loc}}^{\infty}\left(\mathbb{R} ; \mathbb{R}^{n}\right)$. There exists $\alpha=$ $\alpha(m, n) \in(0,1)$ such that $u \in L_{t, \text { loc }}^{\infty} C_{x}^{2, \alpha}\left(\mathbb{R}^{n} \times(0,+\infty)\right)$ and

$$
\underset{t>0}{\operatorname{ess} \sup } t\|\nabla u(\cdot, t)\|_{L^{\infty}\left(\mathbb{R}^{n}\right)}+t^{2}\left\|\nabla^{2} u(\cdot, t)\right\|_{L^{\infty}\left(\mathbb{R}^{n}\right)}+t^{2+\alpha}\left[\nabla^{2} u(\cdot, t)\right]_{C^{\alpha}\left(\mathbb{R}^{n}\right)} \lesssim_{m} 1 .
$$

Proof. The estimate

$$
t^{\alpha}[u(\cdot, t)]_{C^{\alpha}\left(\mathbb{R}^{n}\right)}{ }_{m} 1
$$

follows from a direct application of the $L_{x}^{\infty} \rightarrow C_{x}^{\alpha}$ smoothing estimates developed by Silvestre in [39, Theorem 1.1] and [38] for bounded 'solutions' of non-local drift-diffusion equations

$$
\partial_{t} u+b \cdot \nabla u+\Lambda u=g
$$

where $b, g$ are bounded. Notably, $b$ may be large and not necessarily divergence free. In our situation, $b(x, t)=f^{\prime}(u(x, t))$ and $g=0$. The notion of 'solution' is in quotation marks because, here, $b$ is allowed to be discontinuous, so the notion of viscosity solution may not be directly applicable. ${ }^{7}$ To employ Silvestre's estimates rigorously, one may mollify the initial data, argue at the level of classical solutions, and pass to the limit.

To bootstrap $C_{x}^{\alpha} \rightarrow C_{x}^{1, \alpha}$, we apply linear estimates due to Silvestre in [40, Theorem 1.1] for the drift-diffusion equation (2.20). It is also possible to proceed more directly, as in [11, Appendix B] or in [16,17]. Since $b=f^{\prime}(u)$ is $\alpha$-Hölder continuous in $\mathbb{R}^{n} \times(1 / 2,1)$ with bounds depending only on $m$, Theorem 1.1 in [40] gives

$$
\|u\|_{L_{t}^{\infty} C_{x}^{1, \alpha}\left(\mathbb{R}^{n} \times(1 / 2,1)\right)} \lesssim_{m} 1 .
$$

Hence, $b=f^{\prime}(u)$ satisfies the same bounds. Next, we apply $\partial_{k}, 1 \leq k \leq n$, to the PDE. This gives

$$
\partial_{t} \partial_{k} u+\Lambda \partial_{k} u+b \cdot \nabla \partial_{k} u=-\partial_{k} b \cdot \nabla u .
$$

We regard $g=-\partial_{k} b \cdot \nabla u$ as a forcing term belonging to $L_{t}^{\infty} C_{x}^{\alpha}\left(\mathbb{R}^{n} \times(1 / 2,1)\right)$. Finally, Theorem 1.1 in [40] gives

$$
\left\|\partial_{k} u\right\|_{L_{t}^{\infty} C_{x}^{1, \alpha}\left(\mathbb{R}^{n} \times(3 / 4,1)\right)} \lesssim_{m} 1 .
$$

Scaling invariance gives the sharp dependence on $t$. One could also proceed to higher derivatives.

\footnotetext{
${ }^{7}$ This is discussed in Section 5 of Silvestre's paper [39], see also [40, Section 3]. Silvestre mentions that, if viscosity solutions are unavailable, then one may justify the estimates at the level of the vanishing viscosity approximation

$$
\partial_{t} u^{\varepsilon}+b^{\varepsilon} \cdot \nabla u^{\varepsilon}+\Lambda u^{\varepsilon}=\varepsilon \Delta u^{\varepsilon}
$$

with $\varepsilon \rightarrow 0^{+}$. In principle, this is possible. However, in our setting, the construction in [3] was by an operator splitting method, rather than regularization by $\varepsilon \Delta u^{\varepsilon}$, so we argue differently.
} 
Consider the linear PDE

$$
\partial_{t} u+\Lambda u+b \cdot \nabla u+c u=0
$$

where $b \in L_{t}^{\infty} C_{x}^{1, \alpha}\left(Q_{1}\right)$ and $c \in L_{t}^{\infty} C_{x}^{\alpha}\left(Q_{1}\right)$ with $\|b\|_{L_{t}^{\infty} C_{x}^{1, \alpha}\left(Q_{1}\right)}+\|c\|_{L_{t}^{\infty} C_{x}^{\alpha}\left(Q_{1}\right)} \leq M$. Here, $Q_{T}=\mathbb{R}^{n} \times(0, T)$.

Proposition 2.5 (Fundamental solution estimates). There exists a continuous function $\Gamma=\Gamma(x, t ; y, s), x, y \in \mathbb{R}^{n}$ and $0 \leq s<t \leq 1$, satisfying the following properties:

- (Pointwise upper and lower bounds) For all $0 \leq S \leq s<t \leq T \leq 1$,

$$
C_{0}^{-1} P(x, t ; y, s) \leq \Gamma(x, t ; y, s) \leq C_{0} P(x, t ; y, s),
$$

where $C_{0}=C_{0}(T-S, M)>0$ and $P$ is the Poisson kernel.

- (Maximum principle) If $c \equiv 0$, then

$$
\int_{\mathbb{R}^{n}} P(x, t ; y, s) d y=1 .
$$

- (Representation formula) If $w \in L_{t, x}^{\infty}\left(Q_{1}\right)$, with $w \in L_{t, \text { loc }}^{\infty} C_{x}^{1, \beta}\left(Q_{1}\right)$ for some $\beta \in$ $(0,1)$, is a solution of $(2.25)$ on $Q_{1}$ and $w(\cdot, t) \stackrel{*}{\rightarrow} w_{0}$ in $L^{\infty}\left(\mathbb{R}^{n}\right)$ as $t \rightarrow 0^{+}$, then

$$
w(x, t)=\int_{\mathbb{R}^{n}} \Gamma(x, t ; y, 0) w_{0}(y) d y .
$$

Solutions given by the representation formula belong to the above class.

Proposition 2.5 was obtained in the paper [43] of Xie and Zhang by E. E. Levi's parametrix method, except for uniqueness, which we sketch below. In [43], the authors work with more general assumptions: $b$ in the subcritical space $L_{t}^{\infty} C_{x}^{\alpha}\left(Q_{1}\right)$ and $c$ in a critical Kato space.

Proof of uniqueness. Let $u_{0} \in L^{2}\left(\mathbb{R}^{n}\right)$. Define

$$
v(x, t)=\int_{\mathbb{R}^{n}} \Gamma(x, t ; y, 0) u_{0}(y) d y .
$$

Let $L=\Lambda+b \cdot \nabla+c$ and $L^{*}=\Lambda-b \cdot \nabla+(c-\operatorname{div} b)$. Under our additional regularity assumptions, it is possible to show that $v$ is a weak solution ${ }^{8}$ of the PDE in the sense that

$$
\iint v(x, t)\left(-\partial_{t}+L^{*}\right) \varphi d x d t=0
$$

for all $\varphi \in C_{0}^{\infty}\left(\mathbb{R}^{n} \times(0,1)\right)$. Additionally, we have that $v \in L_{t}^{\infty} L_{x}^{2}\left(Q_{1}\right)$ and $v \in L_{t, \text { loc }}^{2} H_{x}^{1 / 2}\left(\mathbb{R}^{n} \times(0,1]\right)$, among many other spaces, and $v(\cdot, t) \rightarrow u_{0}$ in $L^{2}\left(\mathbb{R}^{n}\right)$ as

\footnotetext{
${ }^{8}$ Due to the quite general conditions in [43], the authors avoided classical solutions and space-time distributional solutions. Instead, they connect the fundamental solution to the PDE via the 'generator' notion.
} 
$t \rightarrow 0^{+}$. This follows from the pointwise upper bounds of the fundamental solution and its first derivatives (see [43, Theorem 1.1(v)]) and the convergence result in [43, Theorem 1.1 (ii)]. One may show, via energy estimates, that the above solution is unique in its class and, additionally, belongs to $C\left([0,1] ; L^{2}\left(\mathbb{R}^{n}\right)\right) \cap L_{t}^{2} H_{x}^{1 / 2}\left(Q_{1}\right){ }^{9}$

Assume now that $u_{0} \in L^{1} \cap L^{\infty}\left(\mathbb{R}^{n}\right)$. Then the above solution $v$ also belongs to $L_{t}^{\infty} L_{x}^{1} \cap L_{t, x}^{\infty}\left(Q_{1}\right)$. By uniqueness within the energy class, the solution $v$ may be obtained by vanishing viscosity:

$$
\partial_{t} u^{\varepsilon}+\Lambda u^{\varepsilon}+b \cdot \nabla u^{\varepsilon}+c u^{\varepsilon}=\varepsilon \Delta u^{\varepsilon} .
$$

According to Silvestre's estimates, we have that $v \in L_{t, \text { loc }}^{\infty} C_{x}^{1, \alpha}\left(\mathbb{R}^{n} \times(0,1]\right)$ for some $\alpha \in(0,1)$ with estimates depending only on $\left\|u_{0}\right\|_{L^{\infty}\left(\mathbb{R}^{n}\right)}$ and the coefficients. By approximation, we have that when $u_{0} \in L^{\infty}\left(\mathbb{R}^{n}\right), v$ satisfies the same a priori estimates.

We now demonstrate the following uniqueness theorem by duality:

If $u \in L_{t, \mathrm{loc}}^{\infty} C_{x}^{1, \alpha}\left(\mathbb{R}^{n} \times(0,1]\right)$ is a solution of the linear PDE $(2.25)$ with $u(\cdot, t) \stackrel{*}{\rightarrow} 0$ in $L^{\infty}\left(\mathbb{R}^{n}\right)$ as $t \rightarrow 0^{+}$, then $u \equiv 0$. $^{10}$

Let $T \in(0,1)$ and $\psi_{0} \in L^{1} \cap L^{\infty}\left(\mathbb{R}^{n}\right)$. The above analysis demonstrated that there exists $\psi \in L_{t}^{\infty} L_{x}^{1} \cap L_{t, x}^{\infty}\left(Q_{T}\right)$ with $\psi \in L_{t, \text { loc }}^{\infty} C_{x}^{1, \alpha}\left(\mathbb{R}^{n} \times[0, T)\right)$ and satisfying the adjoint problem

$$
-\partial_{t} \psi+L^{*} \psi=0
$$

with $\psi(T)=\psi_{0}$. Let $0<t_{0}<t_{1}<T$ and $R, \varepsilon>0$. Let $\chi \in C_{0}^{\infty}\left(B_{2}\right)$ with $\chi \equiv 1$ on $B_{1}$ and $\chi_{R}=\chi(x / R)$. Let $\varphi_{\varepsilon}^{t_{0}, t_{1}}$ be a mollification of the indicator function $\mathbf{1}_{\left(t_{0}, t_{1}\right)}$ at scale $\varepsilon \ll 1$. We test (2.25) against $\psi \chi_{R} \varphi_{\varepsilon}^{t_{0}, t_{1}}$ and omit $t_{0}, t_{1}, R, \varepsilon$ from the notation as convenient:

$$
\begin{aligned}
& \iint \underbrace{\partial_{t} u+L u}_{=0} \psi \chi \varphi d x d t=\iint \underbrace{-\partial_{t} \psi+L^{*} \psi}_{=0} u \varphi d x d t \\
& \quad+\iint\left(-\partial_{t} \varphi\right) \chi u \psi+\varphi(-b \cdot \nabla \chi) u \psi+\varphi u[\Lambda, \chi] \psi d x d t .
\end{aligned}
$$

Upon sending $\varepsilon \rightarrow 0^{+}$, we have

$$
\begin{gathered}
\int \chi_{R} u\left(x, t_{1}\right) \psi\left(x, t_{1}\right) d x-\int \chi_{R} u\left(x, t_{0}\right) \psi\left(x, t_{0}\right) d x \\
=\int_{t_{0}}^{t_{1}} \int_{\mathbb{R}^{n}} b \cdot \nabla \chi_{R} u \psi+u\left[\Lambda, \chi_{R}\right] \psi d x d t
\end{gathered}
$$

for a.e. $t_{0}, t_{1} \in(0,1)$. Moreover, (2.34) is valid for all $t_{0}, t_{1} \in[0, T]$, since $u:[0,1] \rightarrow$ $L^{\infty}\left(\mathbb{R}^{n}\right)$ is weak-* continuous and $\psi \in C\left([0, T] ; L^{2}\left(\mathbb{R}^{n}\right)\right)$. We focus on $t_{0}=0$ and

\footnotetext{
${ }^{9}$ It is important for the energy estimates that $b \in L_{t}^{\infty} C_{x}^{1 / 2}\left(Q_{1}\right)$.

${ }^{10}$ This argument is modeled off a similar argument in [2] by the first author and Zachary Bradshaw.
} 
$t_{1}=T$. First, we recall the following estimate for the Calderón commutator:

$$
\left\|\left[\Lambda, \chi_{R}\right] \psi\right\|_{L^{p^{\prime}}\left(\mathbb{R}^{n}\right)} \lesssim_{p} R^{-1}\|\psi\|_{L^{p^{\prime}}\left(\mathbb{R}^{n}\right)} \text { for all } p \in(1,+\infty) .
$$

Additionally, for $|x| \geq 2 R$, we have

$$
\begin{aligned}
\left|\left[\Lambda, \chi_{R}\right] \psi(x, t)\right| & =c_{n}\left|\int_{\mathbb{R}^{n}} \frac{\chi_{R}(y)}{|x-y|^{n+1}} \psi(y, t) d y\right| \\
& \lesssim_{p}|x|^{-(n+1)+n / p}\|\psi(\cdot, t)\|_{L^{p^{\prime}}\left(\mathbb{R}^{n}\right)}
\end{aligned}
$$

Hence,

$$
\int_{B_{2 R}^{c}}\left|\left[\Lambda, \chi_{R}\right] \psi(x, t)\right| d x \lesssim R^{-1+n / p}\|\psi(\cdot, t)\|_{L^{p^{\prime}}\left(\mathbb{R}^{n}\right)} .
$$

By Hölder's inequality and the above two estimates on $\left[\Lambda, \chi_{R}\right] \psi$, we have

$$
\left|\int_{0}^{T} \int_{B_{2 R} \cup B_{2 R}^{c}} u\left[\Lambda, \chi_{R}\right] \psi d x d t\right| \lesssim_{p} R^{-1+n / p}\|u\|_{L_{t, x}^{\infty}\left(Q_{1}\right)}\|\psi\|_{L_{t}^{\infty} L_{x}^{p^{\prime}}\left(Q_{1}\right)} \rightarrow 0
$$

as $R \rightarrow+\infty$ when $p>n$. The term containing $b \cdot \nabla \chi_{R}$ is $O\left(R^{-1}\right)$, since $b, u \in L_{t, x}^{\infty}\left(Q_{1}\right)$ and $\psi \in L_{t}^{\infty} L_{x}^{1}\left(Q_{T}\right)$. Hence, (2.34) becomes

$$
\int u(x, T) \psi_{0} d x=0
$$

for all $T \in(0,1)$ and $\psi_{0} \in L^{1} \cap L^{\infty}\left(\mathbb{R}^{n}\right)$. Therefore, $u \equiv 0$ on $Q_{1}$.

\section{Proof of main results}

\subsection{Proof of Theorem 1.1}

Let $u_{0} \in L^{\infty}$ and $u$ be the corresponding entropy solution. For each $v_{0}$, we consider the solution $\tilde{u}=u+v$ with initial data $\widetilde{u_{0}}=u_{0}+v_{0} \in L^{\infty}$.

Let $m>0$ and $\left\|u_{0}\right\|_{L^{\infty}},\left\|\widetilde{u_{0}}\right\|_{L^{\infty}} \leq m$.

We prove continuity with respect to $v_{0}$.

Proposition 3.1 (Continuity estimate). Let $1 \leq p \leq q \leq+\infty$. If $v_{0} \in L^{p}\left(\mathbb{R}^{n}\right)$, we have

$$
\|v(\cdot, t)\|_{L^{q}\left(\mathbb{R}^{n}\right)} \lesssim_{m, p, q} t^{\frac{n}{q}-\frac{n}{p}}\left\|v_{0}\right\|_{L^{p}\left(\mathbb{R}^{n}\right)}
$$

Proof. By scaling invariance, it is enough to demonstrate (3.1) with $t=1$.

Step 1. Propagation of localization. First, we demonstrate that, for all $t \in(0,1 / 2]$, we have

$$
\|v(\cdot, t)\|_{\ell_{k}^{p} L_{x}^{1}\left(\mathbb{R}^{n}\right)} \lesssim_{m, p}\left\|v_{0}\right\|_{\ell_{k}^{p} L_{x}^{1}\left(\mathbb{R}^{n}\right)}
$$


Using Proposition 2.1 (controlled speed of propagation), we have

$$
\begin{aligned}
\int_{\square(k)}|v(t, x)| d x & \leq \int_{B(k, \sqrt{2 n} / 2)}|v(x, t)| d x \\
& \leq \int_{B(k, \sqrt{2 n} / 2+L t)} P *\left|v_{0}\right|(x) d x \\
& =\sum_{|j| \leq R} \int_{\square(k+j)} P *\left|v_{0}\right|(x) d x,
\end{aligned}
$$

where $j \in \mathbb{Z}^{n}$ and $R=R(n, L)>0$. We apply $\|\cdot\|_{\ell_{k}^{p}\left(\mathbb{Z}^{n}\right)}$ to each side of (3.3). By the triangle inequality, and since the sum in $j$ has only finitely many boxes, we have

$$
\|v(\cdot, t)\|_{\ell_{k}^{p} L_{x}^{1}\left(\mathbb{R}^{n}\right)} \lesssim_{R}\left\|P *\left|v_{0}\right|\right\|_{\ell_{k}^{p} L_{x}^{1}\left(\mathbb{R}^{n}\right)} .
$$

Now Lemma 2.3 (smoothing for the heat equation) with $q_{1}=q_{2}=1$ gives (3.2).

Step 2. Smoothing. Second, we demonstrate that, for all $t \in(3 / 4,1]$, we have

$$
\|v(\cdot, t)\|_{L^{q}\left(\mathbb{R}^{n}\right)} \lesssim m, p, q\|v(\cdot, 1 / 2)\|_{\ell_{k}^{p} L_{x}^{1}\left(\mathbb{R}^{n}\right)} .
$$

By Proposition 2.4 (regularity), $u$ and $\tilde{u}$ belong to $L_{t}^{\infty} C_{x}^{2, \alpha}\left(\mathbb{R}^{n} \times(1 / 2,1)\right)$ with bounds depending only on $m$. Hence, $v=\tilde{u}-u$ belongs to the same space. Let $w(\cdot, t)=$ $v(\cdot, t+1 / 2)$ when $t \in(0,1 / 2]$. Let $w_{0}=v(\cdot, 1 / 2)$. Then

$$
\partial_{t} w+\operatorname{div}(g(x, t) w)+\Lambda w=0,
$$

where

$$
g(x, t)=\frac{f(\tilde{u})-f(u)}{\tilde{u}-u}
$$

and

$$
\|g\|_{L_{t}^{\infty} C_{x}^{1, \alpha}\left(\mathbb{R}^{n} \times(0,1 / 2)\right)} \lesssim_{m} 1 .
$$

Therefore, we may use the representation formula from Proposition 2.5 (fundamental solution estimates):

$$
w(x, t)=\int_{\mathbb{R}^{n}} \Gamma(x, t ; y, 0) w_{0}(y) d y .
$$

In particular, the pointwise upper bound in Proposition 2.5 gives

$$
|w(x, t)| \lesssim_{m} \int_{\mathbb{R}^{n}} P(x-y, t)\left|w_{0}\right|(y) d y .
$$

Then Lemma 2.3 (smoothing for the heat equation) with $q_{1}=1$ and $q_{2}=q$, along with the embedding $\ell_{k}^{p} L_{x}^{q}\left(\mathbb{R}^{n}\right) \hookrightarrow L^{q}\left(\mathbb{R}^{n}\right)$, gives (3.5).

Finally, we combine (3.2) and (3.5) to complete the proof of Proposition 3.1.

When $v_{0} \in L^{1}\left(\mathbb{R}^{n}\right)$, the propagation of localization comes 'for free' from the $L^{1}$ contraction property, which was shown in [3]. 
Proof of Theorem 1.1. Our goal is to demonstrate the $o_{t \rightarrow+\infty}(1)$ improvement over the conclusion of Proposition 3.1 (continuity estimate) when $p>1$. We approximate $v_{0}$ strongly in $L^{p}\left(\mathbb{R}^{n}\right)$ by $v_{0}^{(k)}$ belonging to $L^{1} \cap L^{\infty}\left(\mathbb{R}^{n}\right)$ and satisfying the decay condition (1.5), $\left|v_{0}^{(k)}\right| \leq\left|v_{0}\right|$, and $\left\|v_{0}^{(k)}\right\|_{L^{\infty}\left(\mathbb{R}^{n}\right)} \leq 2 m$. Let $v^{(k)}$ be the solution corresponding to the initial data $v_{0}^{(k)}$. The $o_{t \rightarrow+\infty}(1)$ improvement is obvious for $v^{(k)}$, which satisfies a faster decay rate because its initial data belongs to $L^{1}\left(\mathbb{R}^{n}\right)$. Next, the triangle inequality and Proposition 3.1 yield

$$
\begin{aligned}
\|v(\cdot, t)\|_{L^{q}\left(\mathbb{R}^{n}\right)} & \leq\left\|v^{(k)}(\cdot, t)\right\|_{L^{q}\left(\mathbb{R}^{n}\right)}+\left\|v(\cdot, t)-v^{(k)}(\cdot, t)\right\|_{L^{q}\left(\mathbb{R}^{n}\right)} \\
& \lesssim_{m} t^{\frac{n}{q}-\frac{n}{p}} O_{t \rightarrow+\infty}(1) \underbrace{\left\|v_{0}^{(k)}\right\|_{L^{p}\left(\mathbb{R}^{n}\right)}}_{\leq\left\|v_{0}\right\|_{L^{p}\left(\mathbb{R}^{n}\right)}}+t^{\frac{n}{q}-\frac{n}{p}} \underbrace{\left\|v_{0}-v_{0}^{(k)}\right\|_{L^{p}\left(\mathbb{R}^{n}\right)}}_{\rightarrow 0 \text { as } k \rightarrow+\infty} .
\end{aligned}
$$

This completes the proof.

Remark 3.2. We record the following alternative proof, due to Hongjie Dong, of Step 1 in Proposition 3.1, without the $\ell_{k}^{p} L_{x}^{1}$ spaces. Consider the adjoint problem to (1.10),

$$
-\partial_{t} w-b \cdot \nabla w+\Lambda w=0
$$

where

$$
b(x, t)=\int_{0}^{1} f^{\prime}(\lambda \tilde{u}+(1-\lambda) u) d \lambda
$$

is Hölder continuous on $\mathbb{R}^{n} \times[1 / 2,1]$. Let $h=P(\cdot, 1 / 2) * v_{0}$ and $x_{0} \in \mathbb{R}^{n}$. Testing the PDE (1.10) against the fundamental solution $\Gamma$ of the adjoint problem with pole at $\left(x_{0}, 1\right)$, we have

$$
\begin{aligned}
\left|v\left(x_{0}, 1\right)\right| & \stackrel{(2.26)}{\lesssim} \sum_{j=0}^{+\infty} 2^{-j} f_{B_{2^{j}}\left(x_{0}\right)}|v(x, 1 / 2)| d x \\
& \stackrel{(2.3)}{\lesssim} \sum_{j=0}^{+\infty} 2^{-j} f_{B_{2^{j}}\left(x_{0}\right)}|h| d x \lesssim(M h)\left(x_{0}\right),
\end{aligned}
$$

where $M h$ is the maximal function of $h$. One can obtain the $L^{p} \rightarrow L^{p}$ bound and, more generally, weighted estimates, straightforwardly from (3.14).

\subsection{BV convergence and spatial asymptotics}

Proof of Theorem 1.2. Let $t_{k} \rightarrow+\infty$ with $t_{k} \geq 1$. It will be convenient to work with the rescaled solutions

$$
u^{(k)}(y, s)=u\left(t_{k} y, t_{k} s\right)
$$

with $\omega^{(k)}=\partial_{y} u^{(k)}$. Then

$$
\left\|\omega^{(k)}(\cdot, 1)-\omega^{\mathrm{ss}}(\cdot, 1)\right\|_{L^{1}(\mathbb{R})}=\left\|\omega\left(\cdot, t_{k}\right)-\omega^{\mathrm{ss}}\left(\cdot, t_{k}\right)\right\|_{L^{1}(\mathbb{R})} .
$$


By Proposition 2.4 (regularity) we can bootstrap the decay of $\left\|u^{(k)}(\cdot, 1)-u^{\mathrm{ss}}(\cdot, 1)\right\|_{L^{\infty}(\mathbb{R})}$ given by Theorem 1.1 to get

$$
\left\|\omega^{(k)}(\cdot, 1)-\omega^{\mathrm{ss}}(\cdot, 1)\right\|_{L^{\infty}(B(R))} \rightarrow 0 \quad \text { as } k \rightarrow+\infty
$$

for all $R \geq 1$. Therefore, it suffices to show that there is no mass of $\omega^{(k)}$ escaping to infinity. Let $R \geq L+10$. By Alibaud's BV formula (2.4), and covering $\mathbb{R} \backslash B(R)$ by an appropriate sequence of balls $B\left(x_{0}, 1\right)$, we have

$$
\int_{\mathbb{R} \backslash B(R)}\left|\omega^{(k)}(x, 1)\right| d x \lesssim m \int_{\mathbb{R} \backslash B(R-L)} P(\cdot, 1) *\left|\omega_{0}^{(k)}\right| d x .
$$

It is not difficult to show that the quantity on the right-hand side is $o_{R \rightarrow+\infty}(1)$ uniformly in $k$.

Proof of (1.8). First, we remark that $u^{\text {ss }}$ is monotone because the evolution of $\omega$ preserves its sign. This is true at the level of entropy solutions, as can be seen from their construction by the splitting argument in Alibaud's paper.

In the following, we allow the constant $C$ to depend on $u_{0}=u_{0}^{\text {ss }}$ and $\tilde{u}_{0}$. Let $a, b \in \mathbb{R}$ represent the limits of $u_{0}$ as $x \rightarrow \mp \infty$.

Step 1. Asymptotics for smooth approximation $\tilde{u}$. Let $\tilde{u}_{0} \in C^{\infty}(\mathbb{R})$ with $\tilde{u}_{0} \equiv u_{0}$ outside of $B_{1}$. Let $\tilde{u}$ be the corresponding entropy solution, which may be shown to belong to $L_{t}^{\infty} C_{x}^{2, \alpha}\left(\mathbb{R}^{n} \times(0,1)\right)$ by combining local-in-time well-posedness ${ }^{11}$ with the estimates in Proposition 2.4 (regularity).

By Proposition 2.5 (fundamental solution estimates), we have

$$
\tilde{u}(x, t)-u_{0}(x)=\int_{\mathbb{R}^{n}} P(x, t ; y, 0)\left[\tilde{u}_{0}(y)-u_{0}(x)\right] d y,
$$

since $\int P(x, t ; y, 0) d y=1$ when $c \equiv 0$. Let $x \leq-1$. Hence,

$$
\tilde{u}(x, t)-u_{0}(x)=\underbrace{\int_{B_{1}} P(x, t ; y, 0)\left[\tilde{u}_{0}(y)-u_{0}(x)\right] d y}_{=I_{1}(x)}+\underbrace{(a-b) \int_{y \geq 1} P(x, t ; y, 0) d y}_{=I_{2}(x)} .
$$

Since $\left[\tilde{u}_{0}-u_{0}(x)\right] \mathbf{1}_{B_{1}}$ is compactly supported, we have that $\left|I_{1}(x)\right| \lesssim\langle x\rangle^{-2}$. On the other hand, when $a \neq b$, we have

$$
C^{-1}\langle x\rangle^{-1} \leq \frac{I_{2}(x)}{a-b} \leq C\langle x\rangle^{-1} .
$$

A similar argument holds for $x \geq 1$. The $I_{2}$ term will dominate when $|x| \geq C$.

\footnotetext{
${ }^{11}$ See the expository blog post [42] of Tao on quasilinear well-posedness.
} 
Step 2. Faster decay for the difference $v$. Let $v=u^{\mathrm{ss}}-\tilde{u}$. We will exploit that $v_{0}=$ $v(\cdot, 1)$ is supported in $B_{1}$ to demonstrate

$$
|v(x, 1)| \leq C\langle x\rangle^{-2}
$$

This will complete the proof, since the $I_{2}$ term will dominate $|v|$ when $|x| \geq C$. We follow the scheme of propagation of localization and smoothing as in the proof of Proposition 3.1. Let $k \in \mathbb{Z}$ with $|k| \geq 10$. By Alibaud's formula and the decay of the Poisson kernel, we have

$$
\int_{\square(k)}|v(\cdot, 1 / 2)| d x \leq C \int_{B(k, \sqrt{2} / 2+L)} P(\cdot, 1 / 2) *\left|v_{0}\right| d x \leq C\langle k\rangle^{-2} .
$$

The difference $v$ also satisfies this estimate when $|k|<10$. Next, we consider $w(\cdot, t)=$ $v(\cdot, t+1 / 2)$ and analyze its representation formula when $t \in(1 / 4,1 / 2]$ :

$$
\begin{aligned}
|w(x, t)| \leq \int_{\mathbb{R}} \Gamma(x, t ; y, 0)\left|w_{0}\right| d y & \leq C \sum_{k \in \mathbb{Z}}\langle k\rangle^{-2}\|\Gamma(x, t ; \cdot, 0)\|_{L_{y}^{\infty}(\square(k))} \\
& \leq C \sum_{k \in \mathbb{Z}}\langle k\rangle^{-2}\langle x-k\rangle^{-2} \\
& \leq C\langle x\rangle^{-2} .
\end{aligned}
$$

The proof is complete.

Remark 3.3 (Rough $f$ ). Suppose that $f$ is locally Lipschitz and $n \geq 1$. It is possible to show that, for each $R>0$, we have

$$
\left\|u-u^{\mathrm{ss}}\right\|_{L^{\infty}(B(R t))} \rightarrow 0 \quad \text { as } t \rightarrow+\infty
$$

That is, $u$ converges to $u^{\text {ss }}$ locally uniformly in self-similar coordinates $y=x / t, s=$ $\log \left(t / t_{0}\right)$ where $t_{0}>0$ is a reference time. Indeed, consider any sequence of rescaled solutions $u^{(k)}$ as above. Since $u_{0}^{(k)} \rightarrow u_{0}^{\text {ss }}$ in $L_{\text {uloc }}^{1}\left(\mathbb{R}^{n}\right)$, Alibaud's formula (2.3) gives that $u^{(k)}(\cdot, 1)$ converges in $L_{\text {uloc }}^{1}\left(\mathbb{R}^{n}\right)$ to $u^{\text {ss }}(\cdot, 1)$. By the a priori Hölder estimates $(2.19)$ and the Ascoli-Arzelá theorem, ${ }^{12} u^{(k)}(\cdot, 1)$ converges in $L_{\mathrm{loc}}^{\infty}\left(\mathbb{R}^{n}\right)$, and its limit must be $u^{\mathrm{ss}}(\cdot, 1)$.

If $n=1$ and $u_{0} \in \mathrm{BV}(\mathbb{R})$, then we may choose $R=+\infty$ in (3.25), since the $\mathrm{BV}(\mathbb{R})$ norm manages the behavior in $L^{\infty}\left(\mathbb{R} \backslash B_{R}\right)$ for $R \gg 1$ according to Alibaud's BV formula (2.4). If $f \in C_{\text {loc }}^{1, \alpha}(\mathbb{R})$, then it is possible to upgrade to $\mathrm{BV}(\mathbb{R})$ convergence, since Silvestre's estimates in [40] allow the solution to be bootstrapped from $C_{x}^{\alpha}(\mathbb{R})$ to $C_{x}^{1, \alpha}(\mathbb{R})$.

\footnotetext{
${ }^{12}$ To justify (2.19) with Lipschitz $f$, one could mollify $f$ or apply a parabolic regularization $\varepsilon \Delta u^{\varepsilon}$, justify the estimates at the regularized level, and pass to the limit.
} 
Acknowledgments. DA thanks Vladimír Šverák for mentioning this problem and helpful discussions. We thank the referee for his/her work on the paper. We also thank Montie Avery, Vlad Vicol, and Jan Burczak for comments on a preliminary version, and Hongjie Dong for the alternative proof in Remark 3.2.

Funding. DA was supported by the NDSEG Fellowship and the NSF Postdoctoral Fellowship Grant No. 2002023. RB was supported by the NSF Graduate Fellowship Grant No. 1839302.

\section{References}

[1] F. Achleitner and Y. Ueda, Asymptotic stability of traveling wave solutions for nonlocal viscous conservation laws with explicit decay rates. J. Evol. Equ. 18 (2018), no. 2, 923-946 Zbl 06932128 MR 3820428

[2] D. Albritton and Z. Bradshaw, Non-decaying solutions to the critical surface quasi-geostrophic equations with symmetries. 2020, arXiv:2011.10856

[3] N. Alibaud, Entropy formulation for fractal conservation laws. J. Evol. Equ. 7 (2007), no. 1, 145-175 Zbl 1116.35013 MR 2305729

[4] N. Alibaud, J. Droniou, and J. Vovelle, Occurrence and non-appearance of shocks in fractal Burgers equations. J. Hyperbolic Differ. Equ. 4 (2007), no. 3, 479-499 Zbl 1144.35038 MR 2339805

[5] N. Alibaud, C. Imbert, and G. Karch, Asymptotic properties of entropy solutions to fractal Burgers equation. SIAM J. Math. Anal. 42 (2010), no. 1, 354-376 Zbl 1225.35026 MR 2607346

[6] M. Beck and C. E. Wayne, Using global invariant manifolds to understand metastability in the Burgers equation with small viscosity. SIAM J. Appl. Dyn. Syst. 8 (2009), no. 3, 1043-1065 Zbl 1201.35044 MR 2551255

[7] P. Biler, T. Funaki, and W. A. Woyczynski, Fractal Burgers equations. J. Differential Equations 148 (1998), no. 1, 9-46 Zbl 0911.35100 MR 1637513

[8] Z. Bradshaw and T.-P. Tsai, Local energy solutions to the Navier-Stokes equations in Wiener amalgam spaces. SIAM J. Math. Anal. 53 (2021), no. 2, 1993-2026 MR 4242337

[9] J. Bricmont, A. Kupiainen, and G. Lin, Renormalization group and asymptotics of solutions of nonlinear parabolic equations. Comm. Pure Appl. Math. 47 (1994), no. 6, 893-922 Zbl 0806.35067 MR 1280993

[10] J. Burczak and R. Granero-Belinchón, Critical Keller-Segel meets Burgers on $\mathbb{S}^{1}$ : large-time smooth solutions. Nonlinearity 29 (2016), no. 12, 3810-3836 Zbl 1362.35318 MR 3580331

[11] L. A. Caffarelli and A. Vasseur, Drift diffusion equations with fractional diffusion and the quasi-geostrophic equation. Ann. of Math. (2) 171 (2010), no. 3, 1903-1930 MR 2680400

[12] C. H. Chan and M. Czubak, Regularity of solutions for the critical $N$-dimensional Burgers' equation. Ann. Inst. H. Poincaré Anal. Non Linéaire 27 (2010), no. 2, 471-501 Zbl 1189.35354 MR 2595188

[13] C. H. Chan, M. Czubak, and L. Silvestre, Eventual regularization of the slightly supercritical fractional Burgers equation. Discrete Contin. Dyn. Syst. 27 (2010), no. 2, 847-861 Zbl 1194.35320 MR 2600693 
[14] I.-L. Chern and T.-P. Liu, Convergence to diffusion waves of solutions for viscous conservation laws. Comm. Math. Phys. 110 (1987), no. 3, 503-517 Zbl 0635.76071 MR 891950

[15] A. Chmaj, Existence of travelling waves in the fractional Burgers equation. Bull. Aust. Math. Soc. 97 (2018), no. 1, 102-109 Zbl 1380.65219 MR 3744871

[16] P. Constantin, A. Tarfulea, and V. Vicol, Long time dynamics of forced critical SQG. Comm. Math. Phys. 335 (2015), no. 1, 93-141 Zbl 1316.35238 MR 3314501

[17] P. Constantin and V. Vicol, Nonlinear maximum principles for dissipative linear nonlocal operators and applications. Geom. Funct. Anal. 22 (2012), no. 5, 1289-1321 Zbl 1256.35078 MR 2989434

[18] M. Dabkowski, Eventual regularity of the solutions to the supercritical dissipative quasigeostrophic equation. Geom. Funct. Anal. 21 (2011), no. 1, 1-13 Zbl 1210.35185 MR 2773101

[19] T. Do, A. Kiselev, L. Ryzhik, and C. Tan, Global regularity for the fractional Euler alignment system. Arch. Ration. Mech. Anal. 228 (2018), no. 1, 1-37 Zbl 1390.35370 MR 3749255

[20] H. Dong, D. Du, and D. Li, Finite time singularities and global well-posedness for fractal Burgers equations. Indiana Univ. Math. J. 58 (2009), no. 2, 807-821 Zbl 1166.35030 MR 2514389

[21] T. Gallay and C. E. Wayne, Global stability of vortex solutions of the two-dimensional NavierStokes equation. Comm. Math. Phys. 255 (2005), no. 1, 97-129 Zbl 1139.35084 MR 2123378

[22] J. Guillod and V. Šverák, Numerical investigations of non-uniqueness for the Navier-Stokes initial value problem in borderline spaces. 2017, arXiv:1704.00560

[23] A. M. Ilín and O. A. Olen̆nik, Behavior of solutions of the Cauchy problem for certain quasilinear equations for unbounded increase of the time. Dokl. Akad. Nauk SSSR 120 (1958), 25-28 MR 0101396

[24] T. Iwabuchi, Global solutions for the critical Burgers equation in the Besov spaces and the large time behavior. Ann. Inst. H. Poincaré Anal. Non Linéaire 32 (2015), no. 3, 687-713 Zbl 1320.35073 MR 3353705

[25] T. Iwabuchi, Analyticity and large time behavior for the Burgers equation and the quasigeostrophic equation, the both with the critical dissipation. Ann. Inst. H. Poincaré Anal. Non Linéaire 37 (2020), no. 4, 855-876 Zbl 1441.35255 MR 4104828

[26] H. Jia and V. Sverak, Are the incompressible 3d Navier-Stokes equations locally ill-posed in the natural energy space? J. Funct. Anal. 268 (2015), no. 12, 3734-3766 Zbl 1317.35176 MR 3341963

[27] C. K. R. T. Jones, R. Gardner, and T. Kapitula, Stability of travelling waves for nonconvex scalar viscous conservation laws. Comm. Pure Appl. Math. 46 (1993), no. 4, 505-526 Zbl 0791.35078 MR 1211740

[28] G. Karch, C. Miao, and X. Xu, On convergence of solutions of fractal Burgers equation toward rarefaction waves. SIAM J. Math. Anal. 39 (2008), no. 5, 1536-1549 Zbl 1154.35080 MR 2377288

[29] Y. J. Kim and A. E. Tzavaras, Diffusive $N$-waves and metastability in the Burgers equation. SIAM J. Math. Anal. 33 (2001), no. 3, 607-633 Zbl 1077.35092 MR 1871412

[30] A. Kiselev, Nonlocal maximum principles for active scalars. Adv. Math. 227 (2011), no. 5, 1806-1826 Zbl 1244.35022 MR 2803787

[31] A. Kiselev and F. Nazarov, A variation on a theme of Caffarelli and Vasseur. Zap. Nauchn. Sem. S.-Peterburg. Otdel. Mat. Inst. Steklov. (POMI) 370 (2009), no. Kraevye Zadachi Matematicheskoŭ Fiziki i Smezhnye Voprosy Teorii Funktsiü. 40, 58-72, 220 MR 2749211 
[32] A. Kiselev, F. Nazarov, and R. Shterenberg, Blow up and regularity for fractal Burgers equation. Dyn. Partial Differ. Equ. 5 (2008), no. 3, 211-240 Zbl 1186.35020 MR 2455893

[33] A. Kiselev, F. Nazarov, and A. Volberg, Global well-posedness for the critical 2D dissipative quasi-geostrophic equation. Invent. Math. 167 (2007), no. 3, 445-453 Zbl 1121.35115 MR 2276260

[34] Y. Maekawa and H. Miura, On fundamental solutions for non-local parabolic equations with divergence free drift. Adv. Math. 247 (2013), 123-191 Zbl 1284.35208 MR 3096796

[35] J. C. Miller and A. J. Bernoff, Rates on convergence to self-similar solutions of Burgers' equation. Stud. Appl. Math. 111 (2003), no. 1, 29-40 Zbl 1141.35451 MR 1985994

[36] D. H. Sattinger, On the stability of waves of nonlinear parabolic systems. Advances in Math. 22 (1976), no. 3, 312-355 Zbl 0344.35051 MR 435602

[37] L. Silvestre, Eventual regularization for the slightly supercritical quasi-geostrophic equation. Ann. Inst. H. Poincaré Anal. Non Linéaire 27 (2010), no. 2, 693-704 Zbl 1187.35186 MR 2595196

[38] L. Silvestre, On the differentiability of the solution to the Hamilton-Jacobi equation with critical fractional diffusion. Adv. Math. 226 (2011), no. 2, 2020-2039 Zbl 1216.35165 MR 2737806

[39] L. Silvestre, Hölder estimates for advection fractional-diffusion equations. Ann. Sc. Norm. Super. Pisa Cl. Sci. (5) 11 (2012), no. 4, 843-855 Zbl 1263.35056 MR 3060702

[40] L. Silvestre, On the differentiability of the solution to an equation with drift and fractional diffusion. Indiana Univ. Math. J. 61 (2012), no. 2, 557-584 Zbl 1308.35042 MR 3043588

[41] L. F. Stokols and A. F. Vasseur, Hölder regularity up to the boundary for critical SQG on bounded domains. Arch. Ration. Mech. Anal. 236 (2020), no. 3, 1543-1591 Zbl 1439.35480 MR 4076071

[42] T. Tao, Quasilinear well-posedness. 2010, https://terrytao.wordpress.com/2010/02/21/quasilinear-well-posedness/ (last visited September 19, 2020)

[43] L. Xie and X. Zhang, Heat kernel estimates for critical fractional diffusion operators. Studia Math. 224 (2014), no. 3, 221-263 Zbl 1309.47054 MR 3294616

[44] K. Zumbrun and P. Howard, Pointwise semigroup methods and stability of viscous shock waves. Indiana Univ. Math. J. 47 (1998), no. 3, 741-871; errata ibid. 51 (2002), no. 4, 10171021 Zbl 0928.35018 MR 1665788

Received 18 October 2020; accepted 31 March 2021.

\section{Dallas Albritton}

Courant Institute of Mathematical Sciences, New York University, New York, NY 10012, USA; daa399@cims.nyu.edu

\section{Rajendra Beekie}

Courant Institute of Mathematical Sciences, New York University, New York, NY 10012, USA; beekie@cims.nyu.edu 